\title{
Possible mechanisms of co-seismic electromagnetic effect
}

\author{
V. V. Surkov ${ }^{1}$ V. A. Pilipenko ${ }^{2}$ (D) A. K. $\operatorname{Sinha}^{3}$
}

Received: 11 July 2017/Accepted: 3 January 2018/Published online: 18 January 2018

(C) Akadémiai Kiadó 2018

\begin{abstract}
We analyze theoretically two possible sources of co-seismic electromagnetic response to the propagation of various types of seismic waves caused either by the electrokinetic phenomena or geomagnetic inductive effect. The differences between these two generation mechanisms have been examined for different types of seismic waves (P, S, and Rayleigh-Love). Theoretical relationships describing the dependence of the co-seismic signal amplitude, polarization and apparent impedance on the earthquake seismic moment and magnitude have been derived as a function of distance. We indicate an observational possibility to discriminate seismo-electrokinetic and seismo-magnetic effects and to estimate their contribution into a recorded co-seismic electromagnetic signal. Magnitudes and polarization of these signals are shown to depend strongly on the type of seismic wave and local crust parameters, such as streaming potential coupling coefficient, conductivity, inhomogeneity, etc. Co-seismic electromagnetic signals, though not directly applied for earthquake prediction, contain a useful information on local crustal phenomena, and can be used to identify "sensitive" zones perspective for the monitoring of precursory electromagnetic disturbances.
\end{abstract}

Keywords Co-seismic effect $\cdot$ Electrokinetic phenomena $\cdot$ Geomagnetic inductive effect $\cdot$ Earthquake $\cdot$ Geophysical prospecting

V. A. Pilipenko

space.soliton@gmail.com

1 National Research Nuclear University MEPhI, Moscow, Russia

2 Institute of Physics of the Earth, Russian Academy of Science, Moscow, Russia

3 Indian Institute of Geomagnetism, Navi Mumbai, India 


\section{Introduction}

Studies of ultra low frequency (ULF) electromagnetic disturbances in seismically active regions have revealed a variety of electromagnetic phenomena related to earthquakes, namely.

- "pre-seismic signals", i.e. irregular or impulsive ULF magnetic pulsations, observed hours to weeks before strong earthquakes (Molchanov and Hayakawa 2008; Surkov and Hayakawa 2014; Pilipenko and Fedorov 2014). This class of events is hard to extract unambiguously from a background noise and thus it is poorly studied so far.

- "co-seismic signals", i.e. electromagnetic signals synchronous with the passage of seismic waves through an observation point after strong far earthquakes (Ivanov 1939, 1940; Martner and Sparks 1959; Eleman 1965; Iyemori et al.1996; Nagao et al. 2000). Also, coherent seismic and electromagnetic fluctuations were recorded after industrial explosions (Anisimov et al. 1985).

These signals are commonly interpreted in terms of two main underlying mechanisms: (a) electrokinetic effect (Fitterman 1978; Frenkel 1944; Martner and Sparks 1959; Pride 1994; Pride and Garambois 2005; Gershenzon et al. 2014; Surkov and Pilipenko 2015), or (b) geomagnetic inductive effect (Knopoff 1955; Kaliski 1960; Guglielmi 1986; Gorbachev and Surkov 1987; Guglielmi and Ruban 1990). The co-seismic phenomena should be distinguished from the long-lasting abnormal offset caused by piezomagnetic (or tectonomagnetic) effects which can occur several weeks around the main shock (Johnston 1978).

Electrokinetic (EK) effect is due to the charge separation in a wet crust. Walls of pores and cracks absorb negative ions from crust fluid, while positive ions remain in the fluid (Frenkel 1944; Bockris and Reddy 1970; Sparnaay 1972). The crust deformations caused by seismic waves induce the crust fluid movement, whereas the movement of fluid ions produces the EK current. The EK current is coupled with the conductivity current in the fluid and encompassing crust. The EK effect was proposed to be responsible for the ULF electromagnetic fluctuations during the final stage of earthquake preparation process owing to an irregular pore fluid flow (Dobrovolsky et al. 1989; Fedorov et al. 2001). The numerical simulation of the co-seismic effect in stratified media has shown that the coseismic signal waveform is sensitive to both the medium structure and seismic source properties (Huang et al. 2015).

Geomagnetic inductive (GMI) perturbations during the seismic wave propagation across an observation site can result from the induction effect owing to oscillations of the conductive crust immersed into the geomagnetic field. Theoretical consideration of the coseismic electromagnetic effect shows that GMI disturbance should spread from a seismic source along a conductive crust in a diffusive way (Surkov 1989; Surkov and Pilipenko 1997; Surkov and Hayakawa 2014). The velocity of the diffusive front can even supersede the seismic wave velocity, thus forming an electromagnetic "precursor" of a seismic wave front (Surkov 1989, 1997; Guglielmi 1991). At larger distances, where the diffusive front is slowed down, GMI disturbance has to propagate together with a seismic wave. Based on the numerical modelling of the co-seismic effect, Molchanov et al. (2001) demonstrated that duration of the GMI perturbation signals has to increase with distance due to dispersion effect in a conductive media.

Observations by Nagao et al. (2000) of co-seismic geoelectric potential changes after $M \geq 5$ class earthquakes that occurred at small epicentral distances, revealed two types of changes: oscillatory response simultaneous with seismic vibrations and offset/decay that 
lasted for about $1 \mathrm{~min}$ after the seismic vibrations ceased. The oscillatory signal was interpreted as a manifestation of electroseismic effect, in which relative motion between solid matrix and pore fluid generates the electric field. The offset/decay change was supposed to be caused by change in the hydrological conditions in shallow subsurface layer under electrodes. Small, yet clear, electric and magnetic signals simultaneous with the $\mathrm{P}$-wave arrival were distinguished from the background noise at several magnetotellutic sites by Honkura et al. (2002). These signals were qualitatively interpreted as a result of "seismic dynamo effect". Surprisingly for authors, they found gradual changes in magnetic components a fraction of second before the arrival of seismic waves. This observational result may be interpreted as an occurrence of the theoretically predicted in Surkov (1989) electromagnetic precursor of the seismic wave front.

The seismo-electric effect is the physical basis of a new method of geophysical prospecting, significantly improving traditional seismic methods. Cross-correlation of seismic and electric signals provides additional important information about oil and gas deposit (Shaidurov et al. 2016).

It is still a debatable issue which of those two effects-GMI or EK, provides a dominant contribution to an observed co-seismic signal. In this paper, we theoretically estimate the expected magnitude and polarization features for two possible sources of the co-seismic electromagnetic effect upon propagation of various types of seismic waves (P, S, Rayleigh or Love). We try to indicate an observational possibility to discriminate these effects and determine their contribution into a recorded co-seismic electromagnetic signal. Co-seismic electromagnetic signals, though not directly applied for earthquake prediction purposes, may contain useful information on local crustal phenomena.

\section{Electrokinetic effect}

The electric field and current can be generated as a result of fluid movement through the pore space of rocks under a gradient of pore pressure. The EK current density in a porous wet sample caused by a gradient of excessive (as compared with the lithostatic pressure) fluid pressure $\nabla P_{f}$ is as follows $\mathbf{j}_{E K}=-\sigma C_{E K} \nabla P_{f}$, where $\sigma$ is the conductivity, and $C_{E K}$ is the EK coefficient. Both coefficients depend on the porosity and permeability of a medium. In the frequency range of typical seismic waves, the displacement current can be neglected as compared with the conductivity current $\sigma \mathbf{E}$. Therefore, the Maxwell's equation comprising both EK and GMI effects is

$$
\nabla \times \mathbf{b}=\mu_{0} \sigma\left(\mathbf{E}-C_{E K} \nabla P_{f}+\mathbf{V} \times \mathbf{B}_{0}\right),
$$

here $\mathbf{b}$ and $\mathbf{E}$ denote the magnetic and electric components of disturbance, $\mu_{0}$ is the magnetic constant, $\mathbf{V}$ is the medium velocity, and $\mathbf{B}_{0}$ is the ambient geomagnetic field. Here and further, we assume that electromagnetic disturbance is weak, i.e. $|\mathbf{b}| \ll\left|\mathbf{B}_{0}\right|$.

In this section we examine the EK effect only and hence the term responsible for the inductive effect, $\mathbf{V} \times \mathbf{B}_{0}$, is omitted in Eq. (1). The electric field is determined by the electric potential $\varphi$ as $\mathbf{E}=-\nabla \varphi$. In a homogeneous boundless medium it follows from Eq. (1) that $\nabla^{2}\left(\varphi+C_{E K} P_{f}\right)=0$, which means that the function $\varphi+C_{E K} P_{f}$ is constant everywhere. Therefore, in the considered approximation $\mathbf{E}=C_{E K} \nabla P_{f}$.

For low-frequency disturbances, a local relationship between the pore fluid pressure $P_{f}$ and the volume deformation of a medium $u_{V}$ is established (Frenkel 1944): 


$$
P_{f}=-\frac{\beta K_{f}}{\alpha} u_{V}, \alpha=1+(\beta-1) \frac{K_{f}}{K_{s}}, \beta=\frac{1}{n}\left(1-\frac{K}{K_{s}}\right) .
$$

here $K_{f}, K_{s}$, and $K$ denote the moduli of a volume compression of a fluid, solid crust structure, and porous dry crust, respectively, and $n$ is the medium porosity.

The solid state physics equation enables one to relate the seismic deformation and velocity with the parameters of a seismic source. We choose the origin of a spherical polar coordinate system in the origin of seismic waves - the earthquake epicenter. The volume deformation of a medium $u_{V}$ caused by a seismic wave is related to the medium displacement $\xi$ as follows (Landau and Lifshitz 1970)

$$
u_{V}=\nabla \cdot \xi=\frac{\partial \xi_{r}}{\partial r}+2 \frac{\xi_{r}}{r}+\frac{1}{r} \frac{\partial \xi_{\theta}}{\partial \theta}+\operatorname{ctg} \theta \frac{\xi_{\theta}}{r}+\frac{1}{r \sin \theta} \frac{\partial \xi_{\varphi}}{\partial \varphi} .
$$

At distances much larger than a seismic fault scale, distributions of the mass velocity and displacement are determined by the tensor of the earthquake seismic moment density, which depends on the size of earthquake focus zone. In the case of simple shear, there are only two non-zero components of the seismic moment tensor $m_{31}=m_{13}=m_{0}$ (Aki and Richards 2002). By the order of magnitude, the seismic tensor components can be estimated as $m=\rho S[\xi] C_{l}^{2}$, where $\rho$ is the medium density, $S$ is the surface of a seismic fault, $[\xi]$ is displacement jump at the fault surface, and $C_{l}$ is the velocity of longitudinal wave. The displacement components can be written in the form

$$
\begin{aligned}
& \xi_{r}=\frac{\sin 2 \theta \cos \phi}{4 \pi \rho r C_{l}^{2}}\left(\frac{1}{C_{l}} \frac{\partial m_{l}}{\partial t}+\frac{4 m_{l}}{r}-\frac{3 m_{t}}{r q}\right), \quad q=\frac{C_{t}^{2}}{C_{l}^{2}}, \\
& \xi_{\theta}=g \cos 2 \theta \cos \phi, \quad \xi_{\phi}=-g \cos \theta \sin \phi, \\
& g=\frac{1}{4 \pi \rho r C_{t}^{2}}\left(\frac{1}{C_{t}} \frac{\partial m_{t}}{\partial t}+\frac{3 m_{t}}{r}-\frac{2 q m_{l}}{r}\right), \quad m_{l, t}=m_{0}\left(t-\frac{r}{C_{l, t}}\right),
\end{aligned}
$$

here the polar axis is directed perpendicular to the plane of a seismic shear, $\theta$ is the angle between the normal to fault surface and direction to an observation site, and $\varphi$ is azimuthal angle measured from the shear direction in the fault plane. Terms in Eq. (4) depend on $m_{l}$ and $m_{t}$ corresponding to a primary longitudinal wave (P-wave) and secondary shear wave (S-wave), propagating with velocities $C_{l}$ and $C_{t}$, respectfully.

To find the volume deformation produced at an observation site by a distant earthquake, we substitute the relationships for medium displacements from Eq. (4) into Eq. (3). In a wave zone, that is at distances $r \gg \lambda$, where $\lambda$ is the seismic wavelength, the term dependent on distance as $r^{-1}$ dominates, because other terms decay fast owing to the small parameter $\lambda / r$. Keeping in Eq. (4) the dominant term only, we arrive at

$$
u_{V}=-\frac{\sin 2 \theta \cos \varphi}{4 \pi \rho r C_{l}^{4}} \frac{\partial^{2}}{\partial t^{2}} m_{0}\left(t-\frac{r}{C_{l}}\right) .
$$

From Eqs. (5) and (2), we find the pore fluid pressure, and subsequently the electric field induced by the EK effect in a seismic wave zone

$$
\mathbf{E}=-\frac{\beta K_{f} C_{E K} \sin 2 \theta \cos \varphi}{4 \pi \alpha \rho r C_{l}^{5}} \frac{\partial^{3}}{\partial t^{3}} m_{0}\left(t-\frac{r}{C_{l}}\right) \hat{\mathbf{r}} .
$$

here $\hat{\mathbf{r}}$ denotes the unit vector along radial direction. The obtained relationship (6) is valid in the laboratory coordinate system. This relationship predicts that only longitudinal P- 
wave can induce the seismo-EK effect. The generated electric field $\mathbf{E}$ is directed approximately along the seismic wave vector. The oscillations of $\mathbf{E}$ and the medium velocity $\mathbf{V}=\partial \xi / \partial t$ do not coincide, because $\mathbf{V}$ is proportional to the second derivative of a seismic moment $m_{0}\left(t-r / C_{l}\right)$. For an order-of-magnitude estimate the Eq. (6) can be reduced to the following form

$$
E_{E K} \sim \frac{\beta K_{f} C_{E K} m_{0}}{4 \pi \alpha \rho r C_{l}^{5} T^{3}}
$$

where $T$ is the seismic wave period. Taking into account that the medium velocity in a Pwave is $V_{m} \sim m_{0} /\left(4 \pi \rho r C_{l}^{3} T^{2}\right)$, the EK electric field estimate can also be rewritten as

$$
E_{E K} \sim \frac{\beta K_{f} C_{E K} V_{m}}{\alpha C_{l}^{2} T}
$$

The estimate for EK response $\mathbf{E}_{E K}$ in Eqs. (7) and (8) must be corrected by adding the term $\mathbf{V} \times \mathbf{B}_{0}$ owing to the measurements in a non-fixed ground coordinate system. This term will be estimated below in Eq. (14).

To estimate an expected magnitude of the co-seismic EK effect we use the empirical dependence of the seismic moment $m_{0}$ on the earthquake magnitude $M$ (Kanamori and Anderson 1975):

$$
m_{0}[\mathrm{~N} \times \mathrm{m}]=10^{1.5 M+9.1} .
$$

Typical parameters of the Earth's upper crust are $n=0.1, K_{s}=2 \mathrm{GPa}, K=0.5 K_{s}$, $K_{f}=0.1 K_{s}, \rho=2 \times 10^{3} \mathrm{~kg} / \mathrm{m}^{3}, C_{l}=5 \mathrm{~km} / \mathrm{s}$, and $C_{E K}=10^{-6}-10^{-8} \mathrm{~V} / \mathrm{Pa}$ (Jouniaux et al. 2000). The EK coefficient $C_{E K}$ may vary in a wide range owing to a large variability of the realistic crust properties, such as porosity, permeability, concentration and mineral composition of crust fluid, etc. For the crust with the above given parameters, the Eq. (7) predicts that a seismic wave with period $T=2 \mathrm{~s}$ at distance $r=10^{3} \mathrm{~km}$ from an earthquake with magnitude $M=7$ can induce the EK electric field $E_{E K} \approx 5-500 \mathrm{nV} / \mathrm{m}$.

\section{Geomagnetic inductive perturbations}

A seismic wave emitted by an earthquake can generate GMI perturbations, which travel together with this wave over long distances. The GMI effect of seismic waves can be described with the Maxwell's quasi-stationary equations. The equation for GMI driven by a seismic wave is deduced from Eq. (1) by applying the curl operation, and substituting electric field $\mathbf{E}$ from the equation $\nabla \times \mathbf{E}=-\partial \mathbf{b} / \partial t$. Assuming $C_{E K}$ is constant, one arrives to the following relationship (Landau and Lifshitz 1960)

$$
\frac{\partial \mathbf{b}}{\partial t}=\frac{1}{\mu_{0} \sigma} \nabla^{2} \mathbf{b}+\nabla \times\left(\mathbf{V} \times \mathbf{B}_{0}\right),
$$

here $\mathbf{b}$ is the perturbation of geomagnetic field $\mathbf{B}_{0}$, and $\mathbf{V}$ is the medium mass velocity in a seismic wave. The crust electrical conductivity $\sigma$ determines the diffusion coefficient of the magnetic field $D=\left(\mu_{0} \sigma\right)^{-1}$. In the vicinity of the elastic seismic wave front the right-hand term in Eq. (10) acts as an external driver. From this equation it follows that GMI disturbance can be produced both by longitudinal and transverse seismic waves. From the 
analysis of this equation solutions for various types of driver function $\mathbf{V}(\mathbf{r}, t)$ can be found (Molchanov et al. 2002; Surkov and Hayakawa 2014).

The characteristic space-time parameters of the problem under consideration are the seismic wave length $\lambda$ and period $T$. Estimating left-hand terms in Eq. (10) as $\partial b / \partial b \sim 2 \pi b / T$, and $\nabla^{2} b \sim 4 \pi^{2} b / \lambda^{2}$, one can find that under the condition $T \ll$ $2 \pi /\left(\mu_{0} \sigma C_{l}^{2}\right)$ the term with time derivative can be neglected as compared with the diffusion term. For typical crustal conductivity $10^{-2}-10^{-3} \mathrm{~S} / \mathrm{m}$, the diffusion limit is valid for $T \ll 2\left(10-10^{2}\right) \mathrm{s}$. The diffusion case corresponds to low values of the Reynolds's number, $\operatorname{Re}_{m}=\mu_{0} \sigma \lambda C_{l} \ll 1$. In this limit the amplitude of GMI perturbation can be estimated as

$$
b_{G M I} \sim \frac{\mu_{0} \sigma \lambda V_{\max } B_{0}}{2 \pi} .
$$

The GMI perturbation can be estimated from the amplitude of the radial component of medium displacement $\xi_{r}$ in the P-wave using $V_{\max } \sim 2 \pi \xi_{r} / T$. It follows from Eq. (4) that $\xi_{r} \sim m_{0}\left(4 \pi \rho r T C_{l}^{3}\right)^{-1}$ whence we obtain

$$
b_{G M I} \sim \frac{\mu_{0} \sigma B_{0} m_{0}}{4 \pi \rho r T C_{l}^{2}} .
$$

For the above crust parameters and estimate (9) of a seismic moment the Eq. (12) predicts that an earthquake with $M=7$ at distance $r=10^{3} \mathrm{~km}$ can produce the GMI disturbance with magnitude $b_{G M I} \approx 2-20 \mathrm{pT}$ in the terrestrial magnetic field $B_{0}=5 \times 10^{-5} \mathrm{~T}$.

It must be taken into account that magnetic and electric sensors in reality oscillate together with crust movement velocity $\mathbf{V}$. Therefore, electromagnetic components $\mathbf{E}^{\prime}, \mathbf{b}^{\prime}$ in this oscillating coordinate system are related to fields in the fixed coordinate system by the non-relativistic transformation $\mathbf{E}^{\prime}=\mathbf{E}+\mathbf{V} \times \mathbf{B}_{0}$ and $\mathbf{b}^{\prime}=\mathbf{b}$. The electric field component of GMI perturbation can be estimated from Eq. (1) in an oscillating coordinate system as

$$
\nabla \times \mathbf{b}^{\prime}=\mu_{0} \sigma \mathbf{E}^{\prime} .
$$

From Eq. (13) it follows that $E_{G M I}^{\prime} \sim 2 \pi b /\left(\mu_{0} \sigma \lambda\right)$. Substituting the magnetic disturbance from Eq. (12) into this relationship, one obtains

$$
E_{G M I}^{\prime} \sim \frac{m_{0} B_{0}}{4 \pi \rho r T^{2} C_{l}^{3}} \sim V_{m} B_{0} .
$$

For the above crust parameters, Eq. (14) gives the estimate of induced electric field $E_{G M I}^{\prime} \approx 0.16 \mathrm{mV} / \mathrm{m}$.

Equation (2) which couples the pore fluid pressure and volume deformation does not take into account the fluid inertia (Frenkel 1944). Therefore, the above estimates of seismoEK response for short-period waves become not very accurate.

In principle, another extreme case is possible, when the parameter $\operatorname{Re}_{m} \gg 1$, which means the "freezing" of the geomagnetic field lines into a medium when its conductivity is very large. This inequality holds, for example, in wet soil with a high electric conductivity or in sea water whose conductivity is approximately 3 orders of magnitude greater than that of the ground. In this limiting case the diffusion term $D \nabla^{2} \mathbf{b}$ in Eq. (10) can be neglected which leads to the following estimate of the GMI disturbance 


$$
b_{G M I} \sim V_{m} B_{0} / C_{l} .
$$

The estimate of the induced electric component remains the same as in Eq. (14).

\section{Co-seismic effect associated with seismic surface waves}

The co-seismic effects owing to surface seismic waves, Rayleigh and Love types, demand a special consideration. Let us first consider a plane quasi-monochromatic Rayleigh wave, propagating along the horizontal axis $x$ in an elastic homogeneous conducting half-space, bounded by a non-conductive atmosphere. The components of the medium velocity in this wave can be presented as (Landau and Lifshitz 1970):

$$
\begin{aligned}
& V_{x}=\frac{V_{m}}{s_{1}}\left\{\exp \left(-k_{R} s_{1} z\right)-\frac{2 s_{1} s_{2}}{1+s_{2}^{2}} \exp \left(-k_{R} s_{2} z\right)\right\} \exp \left[i\left(k_{R} x-\omega t\right)\right], \\
& V_{z}=i V_{m}\left\{\exp \left(-k_{R} s_{1} z\right)-\frac{2}{1+s_{2}^{2}} \exp \left(-k_{R} s_{2} z\right)\right\} \exp \left[i\left(k_{R} x-\omega t\right)\right],
\end{aligned}
$$

here $s_{1}=\left(1-C_{R}^{2} / C_{l}^{2}\right)^{1 / 12.2}$ and $s_{2}=\left(1-C_{R}^{2} / C_{t}^{2}\right)^{1 / 12.2}$, while $\omega, C_{R}$, and $k_{R}=\omega / C_{R}$ respectively are frequency, velocity, and wave number of Rayleigh wave. Axis $z$ is directed downward. Equations (16) and (17) show that this seismic wave is elliptically polarized in the vertical $x-z$ plane.

We first estimate electromagnetic disturbance caused by the EK effect. Using Eqs. (16) and (17), one can calculate a volume deformation of the medium, and then with the help of Eq. (2) the pore fluid pressure $P_{f}$ and electric field $\mathbf{E}=C_{E K} \nabla P_{f}$ can be determined. The final solution (inside the crust $z>0$ ) is as follows (Surkov and Hayakawa 2014):

$$
\mathbf{E}=E_{m} \exp \left[-s_{1} k_{R} z+i\left(k_{R} x-\omega t\right)\right]\left(-i \hat{\mathbf{x}}+s_{1} \hat{\mathbf{z}}\right),
$$

where $\hat{\mathbf{x}}$ and $\hat{\mathbf{z}}$ are unit vectors along $x$ and $z$ axis respectively. The amplitude of electric field disturbance in (18) is

$$
E_{m}=\frac{\beta \omega K_{f} C_{E K} V_{m}}{\alpha s_{1} C_{l}^{2}} .
$$

The potential electric field in the atmosphere $\mathbf{E}=-\nabla \varphi$ can be found from the solution of Laplace's equation $\nabla^{2} \varphi=0$. Taking into account the continuity of the electric potential at $z=0$, one arrives at expression for the electric field in the atmosphere $(z<0)$

$$
\mathbf{E}=-E_{m} \exp \left[k_{R} z+i\left(k_{R} x-\omega t\right)\right](i \hat{\mathbf{x}}+\hat{\mathbf{z}}) .
$$

It is interesting to note that inside the ground electric perturbations given by Eq. (18) have an elliptic polarization with counterclockwise rotation (looking from Fig. 1 along $y$-axis) analogous to the medium velocity components given by Eq. (16), whereas in the atmosphere the electric field has a circular polarization with clockwise rotation. For oppositely propagating Rayleigh wave, the polarization ellipse rotation changes its sense.

Amplitudes of EK response to P-wave given by Eq. (8) and to Rayleigh wave described by Eqs. (19) and (20) look similar. However, there is a substantial difference between them: the amplitudes of mass velocities $V_{m}$ depend on a distance $r$ from a seismic source differently. For large epicentral distances, in P-wave $V_{m} \sim r^{-1}$, whereas in Rayleigh wave 
Fig. 1 Geometry of the considered model. The rotation sense of the induced E-field and seismic velocity $\mathrm{V}$ is indicated by arrows

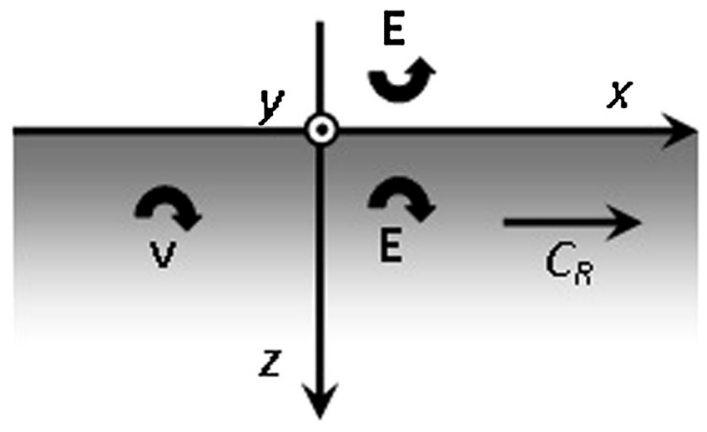

$V_{m} \sim r^{-1 / 2}$. Thus, at large distances the Rayleigh wave contribution to co-seismic effect is significantly larger than that of the P-wave.

Now we analyze GMI disturbances induced by a Rayleigh wave, neglecting EK current. In this case, the magnetic component of a disturbance is described by the quasi-stationary Maxwell Eq. (10), driven by the crust velocities in a seismic wave determined by Eqs. (16) and (17). The magnetic disturbance in the atmosphere is determined by the Laplace equation $\nabla^{2} \mathbf{b}=0$, whereas at the atmosphere-ground interface $(z=0)$ the standard boundary conditions for magnetic field and vertical component of electric current hold. The solution in the atmosphere is as follows (Guglielmi 1986; Gorbachev and Surkov 1987):

$$
\begin{gathered}
\mathbf{b}=b_{m}(i \hat{\mathbf{x}}+\hat{\mathbf{z}}) \exp \left[k_{R} z+i\left(k_{R} x-\omega t\right)\right] . \\
\mathbf{E}=\left[E_{m}(i \hat{\mathbf{x}}+\hat{\mathbf{z}})+b_{m} C_{R} \hat{\mathbf{y}}\right] \exp \left[k_{R} z+i\left(k_{R} x-\omega t\right)\right] .
\end{gathered}
$$

Here the following notations have been used

$$
\begin{aligned}
& b_{m}=\frac{V_{m}\left\{\left(s_{1} a_{2}-a_{1}\right) B_{0 x}+i\left(a_{2}-s_{2} a_{1}\right) B_{0 z}\right\}}{D\left(k_{R}+\alpha_{R}\right)}, \quad \alpha_{R, t, l}^{2}=\frac{\omega^{2}}{C_{R, t, l}^{2}}-\frac{i \omega}{D}, \\
& a_{1}=\frac{2 k_{R}\left(s_{2} k_{R}-\alpha_{R}\right)}{\left(1+s_{2}^{2}\right) \alpha_{t}^{2}}, \quad a_{2}=\frac{k_{R}\left(s_{1} k_{R}-\alpha_{R}\right)}{s_{1} \alpha_{l}^{2}}, \\
& E_{m}=V_{m} B_{0 y}\left(\frac{k_{R} \alpha_{R} C_{R}^{2}}{s_{1} \alpha_{l}^{2} C_{l}^{2}}-\frac{i \omega}{D \alpha_{l}^{2}}-\frac{2}{1+s_{2}^{2}}\right),
\end{aligned}
$$

where $B_{0 x}, B_{0 y}$, and $B_{0 z}$ are the geomagnetic field components. Equation (21) shows that a magnetic disturbance in the atmosphere has a circular polarization with clockwise rotation in the vertical plane.

In the limiting case $\operatorname{Re}_{m} \ll 1$ or $T \ll 2 \pi /\left(\mu_{0} \sigma C_{l}^{2}\right)$, the coefficients in Eq. (23) are simplified, as $\alpha_{R, t, l} \approx \omega / C_{R, t, l}$. In addition, we assume that all the geomagnetic field components are of the order of $B_{0}$. Then an estimate of the amplitude of GMI perturbation reduces to the following

$$
b_{G M I} \simeq \eta \frac{V_{\max } C_{R} B_{0}}{\omega D}=\eta \frac{\mu_{0} \sigma \lambda_{R} V_{\max } B_{0}}{2 \pi},
$$

where $\lambda_{R}$ is the Rayleigh wave length. This estimate comprises the dimensionless factor $\eta \sim 1$, dependent on dimensionless parameters $C_{R} / C_{l}, C_{R} / C_{t}$ and geomagnetic field inclination and declination. The geomagnetic effect estimates given by Eqs. (11) and (24) 
for P-wave and Rayleigh wave look similar. However, it must be noticed that the medium velocity amplitudes produced by Rayleigh wave decay with distance slower, $V_{\max } \sim r^{-1 / 2}$, as compared with that by the body $\mathrm{P}$-wave and S-wave, $V_{\max } \sim r^{-1}$.

Analysis of coefficients in Eq. (22) shows that under $\operatorname{Re}_{m} \ll 1$ the inequality $E_{m} \sim$ $V_{m} B_{0} \gg b_{m} C_{R}$ holds (besides an extreme case $B_{0 y}=0$ ). This means that the transverse electric field component $E_{y}$ is weak and can be neglected. Therefore, similar to magnetic components, the electric components of GMI disturbance are circularly polarized in the vertical plane. The amplitude of disturbance, $E_{G M I} \sim V_{m} B_{0}$, is the same as that given by Eq. (14).

The derived relationships enable one to estimate the magnetic and electric effects of seismic waves by an order of magnitude and also they can be used to study their polarization characteristics. In the Love wave the crust displacement is oriented along $y$-axis, perpendicular to the wave propagation direction along the ground surface (Landau and Lifshitz 1970). The co-seismic electromagnetic effect produced by Love wave can be calculated similar to the previous consideration. The GMI signal has approximately circular polarization in the vertical plane parallel to the seismic wave propagation direction. This feature was used by Guglielmi et al. (2006) to reveal weak seismo-magnetic pulsations $(\sim 0.01-0.04 \mathrm{nT})$ induced by Love waves from very distant powerful seismic events $(M>7)$.

\section{Comparison of EK and GMI effects}

Many researchers interpreted co-seismic telluric field disturbances on the basis of EK effect only. To validate this assumption, let us compare the estimate of isolated EK effect given by Eq. (7) to that of GMI effect given by Eq. (13) under the same P-wave parameters

$$
\frac{E_{E K}}{E_{G M P}^{\prime}} \sim \frac{\beta K_{f} C_{E K}}{\alpha B_{0} T C_{l}^{2}} .
$$

Nearly the same relationship stems from the consideration of surface seismic waves. The estimate (25) depends strongly on the crust porosity $n$, which varies in a very wide range. For example, typical values of $n$ for granite and gneiss are $(0.2-6) \times 10^{-3}$, for sandstone 0.04-0.3, and for tuff 0.2-0.3 (Mavko et al. 2009). For the above used parameters and $n=0.003-0.1$, it follows from (25) that $E_{E K} / E_{G M P}^{\prime}<1, \quad$ if $C_{E K}<(4-9) \times 10^{-8} \mathrm{~V} / \mathrm{Pa}$. This critical value of $C_{E K}$ corresponds approximately to the value of streaming potential coefficient of Westerly granite (Morgan et al. 1989). Thus, which mechanism does produce a largest co-seismic effect is very uncertain, because the ratio between these mechanisms depends on specific crust parameters.

Oscillations of the ground surface influence the electric field recordings, and the correction factor $\sim V_{m} B_{0}$ can be comparable to $E_{E K}$. Therefore, a proper seismo-electric signal can be masked by the effect due to the sensor oscillations.

In a homogeneous medium the EK mechanism does not produce any magnetic disturbances above the ground. Magnetic disturbances can emerge near lateral inhomogeneities of streaming potential coupling coefficient $C_{E K}$ and conductivity $\sigma$. In a simple model of two half-spaces with constant coefficients $C_{1}, \sigma_{1}$ and $C_{2}, \sigma_{2}$, separated by a vertical plane, the magnetic disturbance near the interface can be estimated as follows (Fitterman 1979a, b; Gershenzon et al. 2014): 


$$
b_{E K}=\frac{\mu_{0} \sigma_{1} \sigma_{2} P_{f} \Delta C_{E K} f}{2 \pi\left(\sigma_{1}+\sigma_{2}\right)},
$$

here $\Delta C_{E K}=C_{1}-C_{2}$ is the contrast of the streaming potential coupling coefficients, and $f \approx 10-20$ is the geometrical factor. The pore fluid pressure can be estimated by Eq. (2), keeping in mind that a volume deformation in a P-wave is $u_{V} \sim V_{m} / C_{l}$. Substituting $P_{f}$ into (26) and supposing that $\sigma_{1}=\sigma_{2}=\sigma$, we arrive at

$$
b_{E K} \sim \frac{\mu_{0} \sigma \beta K_{f} V_{m} \Delta C_{E K} f}{2 \pi \alpha C_{l}} .
$$

Let us compare the estimate (27) with the estimate for GMI effect from Eq. (11)

$$
\frac{b_{E K}}{b_{G M P}} \sim \frac{\beta K_{f} \Delta C_{E K} f}{2 \alpha T C_{l}^{2} B_{0}} .
$$

Uncertainty of the gradient value $\Delta C_{E K}$ at the interface greatly reduces the accuracy of estimate (28) in the frameworks of the considered model. For the same set of parameters that have been used above ( $n=0.003-0.1$, and $\Delta C_{E K}=10^{-6}-10^{-8} \mathrm{~V} / \mathrm{Pa}$ ), the estimate (28) can vary in a very wide range, from $1.4 \times 10^{-3}$ to 80 .

The above estimates have proven that both mechanisms might be responsible for coseismic effects, which can be detected on the natural electromagnetic noise background. In measurements of co-seismic electric component, the GMI mechanism is dominant for lowporosity high-resistive media like granite. The EK mechanism should dominate in highconductive and fluid-saturated media, especially when these features occur deep in the crust, at least to a depth about the seismic wave length.

In measurements of co-seismic magnetic component, the GMI mechanism is largest in a homogeneous medium. However, near the lateral geoelectric inhomogeneities the EK mechanism becomes significant, too. More precise conclusions can be done only where the specific geoelectric and fluid structure are known.

\section{Discussion}

In general, both EK and GMI mechanisms can interpret qualitatively the occurrence of coseismic electromagnetic signals. In particular, GMI mechanism can interpret even a fine effect (Surkov 1997) — a weak precursory deviations of magnetic field about a second before a seismic wave arrival in an observational site (Honkura et al. 2002). Nonetheless, the modeling of quantitative relationships between seismic wave disturbances, electromagnetic response, and local crust parameters is important and highly needed. Co-seismic electromagnetic signals, though not directly applied for earthquake prediction purposes, contain a useful information on local crustal phenomena, and can be used to identify "sensitive" zones perspective for the monitoring of precursory electromagnetic disturbances. Extensive observational search for earthquake electromagnetic precursory effects revealed that the occurrence of such precursors has a "mozaic" character. This feature may be related to inhomogeneous spatial distribution of local crust parameters, such as porosity, fluid concentration, conductivity, EK coefficient, medium gradient, etc. Thus, a preliminary examination of the co-seismic effects throughout monitoring area may help to identify sites that are most sensitive to the crust pressure variations. 
For practical applications it is important to find methods that would help to identify a responsible mechanism for detected co-seismic signals. In a realistic situation, co-seismic signal can be obscured by background noise, magnetospheric pulsations, seismographic vibrations, etc. Moreover, one of the problematic aspect of the theory is that though earth crust is not homogeneous, but it has been treated as locally homogeneous. The P-wave and Rayleigh wave produce a volume deformation of the crust causing both effects. At the same time, S-wave and Love wave produce transverse deformations only and hence they should not induce the EK mechanism.

A powerful tool to identify the physical nature of an electromagnetic disturbance is the determination of an apparent impedance, that is the ratio between electric and magnetic components (Pilipenko et al. 2003). The apparent impedance of the GMI disturbance excited by a $\mathrm{P}$-wave is as follows

$$
Z_{G M I}=E_{G M I}^{\prime} / b_{G M I} \sim\left(\mu_{0} \sigma C_{l} T\right)^{-1} .
$$

For the given above parameters, we get $Z_{\mathrm{GMI}} \sim 10^{4}-10^{5} \mathrm{~m} / \mathrm{s}$. The measured magnitudes of an apparent impedance is to be compared with the conductive ground impedance $Z_{g}=\sqrt{\mu_{o} \omega / \sigma}$. A noticeable deviation of impedance of detected signals from $Z_{g}$ indicates a non-magnetospheric origin of those signals.

For a laterally homogeneous crust a magnetic component of the co-seismic EK signal must vanish. However, the occurrence of inhomogeneities can result in the appearance of magnetic effect. The estimate for the vertical contact model given by Eq. (26) may be considered as an upper limit of the magnitude of this effect.

The mechanisms of co-seismic effect may be discriminated by the difference of the spectral content and wave forms of the co-seismic signals. For example, the electric field disturbance $\mathbf{E}_{E K}$ for P-wave (6) and surface wave (19) is proportional to the medium acceleration in the epicenter, $\partial^{3} m_{0} / \partial t^{3}$. At the same time, the electric field disturbance owing to the GMI effect is proportional to a medium velocity, $E_{G M I} \sim V_{m} B_{0}$. A phase shift between GMI disturbance and medium velocity depends on many factors, such as crust conductivity and geomagnetic field orientation.

The polarization features of co-seismic signals depend on type of seismic wave. If a seismic wave is produced by a crust shear in the source region, electric EK disturbances $\mathbf{E}_{E K}$ induced by $\mathrm{P}$-wave are oriented in a radial direction parallel to the seismic velocity vector. Magnetic disturbance at large distances from a seismic source has a magneto-dipole character (Surkov and Hayakawa 2014). The electric component $\mathbf{E}_{G M I}$ is to be perpendicular to the plane incorporating the magnetic moment and radius-vector from a seismic source. Therefore, for a direct P-wave a predominance of either longitudinal or perpendicular E-field components of co-seismic response determines the dominance of one or other generation mechanism.

For a Rayleigh wave the electric components in the atmosphere from both mechanisms, $\mathbf{E}_{E K}$ and $\mathbf{E}_{G M I}$, have a circular polarization with clockwise rotation in the vertical plane. The main distinction may exist in the features of magnetic components of the co-seismic response. For both types of seismic waves, Rayleigh and Love, the magnetic components produced by induction mechanism $b_{G M I}$ have a circular polarization with clockwise rotation in the vertical plane parallel to the seismic wave propagation direction. At the same time, magnetic disturbance $b_{E K}$, produced by EK mechanism, has distinct features. It can be observed only above crust regions with lateral inhomogeneities of the streaming potential coupling coefficient and conductivity. Its polarization depends on a structure of inhomogeneous crust, so exact polarization features cannot be theoretically predicted. 


\section{Conclusion}

Our theoretical analysis has shown that in principle the observed magnitudes of co-seismic electromagnetic signals can be interpreted as both the EK effect in a wet crust or the inductive response of a conductive crust. However, because of a large variability of the realistic crust parameters, such as porosity, permeability, conductivity, etc., the estimates made for P- and Rayleigh waves make it difficult to conclude unambiguously which of those mechanisms prevails. With some certainty, one can say that EK effect is weak for shear S-wave and Love wave, which do not produce a deformation in the crust volume.

The electric components for both mechanisms induced by Rayleigh wave have a circular polarization in the atmosphere in the vertical plane. The polarization of the electric components induced by a P-wave may be different: longitudinal for the EK effect and transverse for the GMI perturbation.

A difference between the EK and GMI mechanisms is more evident in the magnetic components of a co-seismic signal. The EK mechanism can produce magnetic response only in an inhomogeneous medium with a strong lateral gradient of the streaming potential coupling coefficient and conductivity. Therefore, polarization features of these disturbances are determined by specific properties of the crust. At the same time, the GMI mechanism can generate magnetic response in a conductive homogeneous half-space. Its magnitude is determined by the media conductivity and the geomagnetic field orientation. GMI disturbance induced by Rayleigh and Love waves has a circular polarization in the vertical plane.

Thus, the considered mechanisms of the co-seismic disturbance generation by seismic waves induce electromagnetic responses with different characteristics, which provide a principal feasibility to discriminate them during realistic observations.

Acknowledgements This study is supported by the Grant No 15-55-45064 from the Russian Foundation for Basic Sciences (VAP, AKS) and by the Competitiveness Program of NRNU MEPhI (VVS). We appreciate useful comments of both reviewers.

\section{References}

Aki K, Richards P (2002) Quantitative Seismology, 2nd edn. University Science Books, Sausalito, p 932

Anisimov SV, Gokhberg MB, Ivanov EA et al (1985) Short period vibrations of the Earth's electromagnetic field due to industrial explosion. Rep USSR Acad Sci 281(3):556-559

Bockris JOM, Reddy AKN (1970) Modern electrochemistry. Plenum, New York, pp 623-843

Dobrovolsky IP, Gershenzon NI, Gokhberg MB (1989) Theory of electrokinetic effects occurring at the final state in the preparation of a tectonic earthquake. Phys Earth Planet Inter 57:144-156

Eleman F (1965) The response of magnetic instruments to earthquake waves. J Geomagn Geoelectr $18: 43-72$

Fedorov E, Pilipenko V, Uyeda S (2001) Electric and magnetic fields generated by electrokinetic processes in a conductive crust. Phys Chem Earth 26:793-799

Fitterman DV (1978) Electrokinetic and magnetic anomalies associated with dilatant regions in a layered earth. J Geophys Res 83:5923-5928

Fitterman DV (1979a) Theory of electrokinetic-magnetic anomalies in a faulted half-space. J Geophys Res 84:6031-6040

Fitterman DV (1979b) Calculations of self-potential anomalies near vertical contacts. Geophysics 44:195-205

Frenkel YAI (1944) On the theory of seismic and seismoelectric phenomena in a moist soil. J Phys (USSR) $8: 230-241$ 
Gershenzon NI, Bambakidis G, Ternovskiy I (2014) Coseismic electromagnetic field due to the electrokinetic effect. Geophysics 79:E217-E229

Gorbachev LP, Surkov VV (1987) Perturbation of an external magnetic field by a Rayleigh surface wave. Magnetohydrodynamics 23:117-125

Guglielmi AV (1986) Excitation of electromagnetic field oscillations by elastic waves in conducting body. Geomagn Aeron 27:467-470

Guglielmi AV (1991) Magnetic structure of the elastic wave front in conductive media. Izv Akad Nauk SSSR Ser Fiz Zemli 4:753-758

Guglielmi AV, Ruban VF (1990) On the theory of the seismomagnetic induction effect. Izv Akad Nauk SSSR Ser Fiz Zemli N5:47-54

Guglielmi A, Hayakawa M, Potapov A, Tsegmed B (2006) Polarization method to detect the co-seismic magnetic oscillations. Phys Chem Earth 31:299-304

Honkura Y, Matsushima M, Oshiman N et al (2002) Small electric and magnetic signals observed before the arrival of seismic wave. Earth Planets Space 54:e9-e12

Huang Q, Ren H, Zhang D, Chen YJ (2015) Medium effect on the characteristics of the coupled seismic and electromagnetic signals. Proc Jpn Acad Ser B Phys Biol Sci 91:17-24

Ivanov AG (1939) Effect of electrization of earth layers by elastic waves passing through them. Proc USSR Acad Sci 24:42-45

Ivanov AG (1940) Seismoelectric effect of the second kind. Proc USSR Acad Sci Ser Geogr Geophys 4:699-726

Iyemori T, Kamei T, Tanaka Y et al (1996) Co-seismic geomagnetic variations observed at the 1995 Hyogoken-Nanbu earthquake. J Geomagn Geoelectr 48:1059-1070

Johnston MJS (1978) Tectonomagnetic effects. Earthq Inf Bull (USGS) 10:82-87

Jouniaux L, Bernard ML, Zamora M, Pozzi JP (2000) Streaming potential in volcanic rocks from Mount Pelée. J Geophys Res 105:8391-8401

Kaliski S (1960) Solution of the equation of motion in a magnetic field for an isotropic body in an infinite space assuming perfect electric conductivity. Proc Vibr Probl 1(3):53-67

Kanamori H, Anderson DL (1975) Theoretical basis of some empirical relations in seismology. Bull Seism Soc Am 65:1073-1095

Knopoff EL (1955) The interaction between elastic waves motion and a magnetic field in electrical conductors. J Geophys Res 60:617-629

Landau LD, Lifshitz EM (1960) Electrodynamics of continuous media. In: Course of theoretical physics, vol 8. Pergamon Press, Oxford

Landau LD, Lifshitz EM (1970) Theory of elasticity. In: Course of theoretical physics, vol 7. Pergamon Press, Oxford

Martner ST, Sparks NR (1959) The electroseismic effect. Geophysics 24:297-308

Mavko G, Mukerji T, Dvorkin T (2014) The rock physics handbook: tools for seismic analysis of porous media, 2nd edn. Cambridge University Press, Cambridge

Molchanov OA, Hayakawa M (2008) Seismo-electromagnetics and related phenomena: history and latest results. TERRAPUB, Tokyo, p 189

Molchanov O, Kulchitsky A, Hayakawa M (2001) Inductive seismo-electromagnetic effect in relation to seismogenic ULF emission. Nat Hazards Earth Syst Sci 1:61-67

Molchanov O, Kulchitsky A, Hayakawa M (2002) ULF emission due to inductive seismo-electromagnetic effect. In: Hayakawa M, Molchanov OA (eds) Seismo-electromagnetics: lithosphere-atmosphereionosphere coupling. TERRARUB, Tokyo, pp 153-162

Morgan FD, Williams ER, Madden TR (1989) Streaming potential properties of Westerly granite with applications. J Geophys Res 94:12449-12461

Nagao T, Orihara Y, Yamaguchi T et al (2000) Co-seismic geoelectric potential changes observed in Japan. Geophys Res Lett 27:1535-1538

Pilipenko V, Fedorov E (2014) Coupling mechanism between geoacoustic emission and electromagnetic anomalies prior to earthquakes. Res Geophys 4:5008. https://doi.org/10.4081/rg.2014.5008

Pilipenko V, Nenovski P, Tanaka H (2003) Detection and discrimination of VLF/ULF seismic-related electromagnetic emissions. Bulg Geophys J 29:13-30

Pride SR (1994) Governing equations for the coupled electromagnetics and acoustics of porous media. Phys Rev B 50:15678-15696

Pride SR, Garambois S (2005) Electroseismic wave theory of Frenkel and more recent developments. J Eng Mech 131:898-907

Shaidurov GYA, Potylitsyn VS, Kudinov DS (2016) Phase-induced polarization method based on processing noise signals of the natural electromagnetic field of the earth. Rus Geol Geophys 57:1371-1376

Sparnaay MJ (1972) The electrical double layer. Pergamon Press, New York, p 415 
Surkov VV (1989) Perturbation of an external magnetic field by a longitudinal acoustic wave. Magnetohydrodynamics 25:145-148

Surkov VV (1997) The electromagnetic precursor of a seismic wave. Geomagn Aeron 37:792-796

Surkov V, Hayakawa M (2014) Ultra and extremely low frequency electromagnetic fields, springer geophysics series, vol XVI. Springer, Berlin, p 486

Surkov VV, Pilipenko VA (1997) Magnetic effects due to earthquakes and underground explosions: a review. Ann Geophys 40(2):227-239. https://doi.org/10.4401/ag-3904

Surkov VV, Pilipenko VA (2015) Estimate of ULF electromagnetic noise caused by a fluid flow during seismic or volcano activity. Ann Geophys 58(6):S0655. https://doi.org/10.4401/ag-6767 